\section{MÉTODO CONVENCIONAL PARA O DIAGNÓSTICO DE MICOSES: UM ESTUDO COMPARATIVO}

\section{CONVENTIONAL METHOD FOR THE DIAGNOSIS OF MYCOSES: A COMPARATIVE STUDY}

\author{
Priscilla Maciel Quatrin 1 , Ânderson Ramos Carvalho², Janaína Scarton ${ }^{3}$, \\ Osmar Luís Magalhães de Oliveira ${ }^{3}$, Alexandre Meneghello Fuentefria ${ }^{1,2}$
}

\section{RESUMO}

Introdução: O método clássico para o diagnóstico de micoses é realizado pelo Exame Micológico Direto (EMD) e cultural, que possibilita a visualização de estruturas fúngicas vegetativas e estruturas reprodutivas, respectivamente. Essa combinação é fundamental para reduzir possíveis erros analíticos e aumentar a precisão do diagnóstico.

Métodos: Com a finalidade de verificar a frequência do EMD e cultural, e comparar seus parâmetros de sensibilidade e especificidade, realizamos uma análise retrospectiva entre janeiro de 2018 e maio de 2020, de 1603 laudos micológicos oriundos de um laboratório de análises clínicas, localizado em Porto Alegre.

Resultados: Após a análise dos laudos observamos que a maioria dos casos apresentaram o EMD negativo com cultura positiva (36,24\%). Na sequência, $30,87 \%$ dos casos foram de amostras negativas e $25,57 \%$ dos laudos foram positivos para ambos os exames. A minoria dos casos $(7,29 \%)$ apresentou o EMD positivo com cultura negativa.

Conclusão: Esta análise revelou que o exame cultural é mais sensível e específico, demonstrando uma maior confiabilidade no diagnóstico. Entretanto, vale ressaltar que a realização dos exames em conjunto, além de reduzir possíveis erros analíticos, proporciona um diagnóstico melhor fundamentado.

Palavras-chave: Exame micológico direto; cultura; diagnóstico micológico

\section{ABSTRACT}

Introduction: The classic method for the diagnosis of mycoses is performed by both direct mycological examination (DME) and culture, which allow the visualization of vegetative and reproductive fungal structures, respectively. This combination is essential to reduce possible analytical errors and increase the accuracy of the diagnosis.

Methods: To assess the frequency of DME and culture, and compare their parameters of sensitivity and specificity, we performed a retrospective analysis of 1603 mycological reports produced between January 2018 and May 2020 in a clinical analysis laboratory in Porto Alegre, southern Brazil.

Results: After analyzing the reports, we observed that most cases presented a negative DME and a positive culture (36.24\%). Subsequently, $30.87 \%$ of the cases were negative for both tests, and $25.57 \%$ were positive for both tests. The minority of cases $(7.29 \%)$ presented a positive DME and a negative culture.

Conclusion: Our analysis revealed that cultural examination is more sensitive and specific, showing greater reliability in the diagnosis. However, it is noteworthy that performing the tests together, in addition to reducing possible analytical errors, provides a more consistent diagnosis.

Keywords: Direct mycological examination; culture; mycological diagnosis
Clin Biomed Res. 2020;40(4):213-217

1 Programa de Pós-Graduação em Microbiologia Agrícola e do Ambiente, Universidade Federal do Rio Grande do Sul. Porto Alegre, RS, Brasil.

2 Programa de Pós-Graduação em Ciências Farmacêuticas, Universidade Federal do Rio Grande do Sul. Porto Alegre, RS, Brasil.

3 Laboratório Mont'Serrat. Porto Alegre, RS, Brasil.

Autor correspondente: Priscilla Maciel Quatrin pri_mq@hotmail.com Universidade Federal do Rio Grande do Sul (UFRGS)

Avenida Ipiranga, $\mathrm{n}^{\circ} 2752$ 90610-000, Porto Alegre, RS, Brasil. 


\section{INTRODUÇÃO}

A prescrição do exame micológico é fundamental para confirmar o diagnóstico de uma micose, mas também para precisamente identificar o agente patogênico, auxiliando assim na decisão de um tratamento com o antifúngico mais adequado. É cosenso mundial que um exame micológico bem conduzido permite orientar a precisa conduta clínica, já que um resultado negativo o redireciona para outras patologias ${ }^{1}$.

A combinação do exame micológico direto (EMD) com a cultura continua sendo o método tradicional de diagnóstico, pois além de possuir um baixo custo, ambos possuem particularidades que influenciam na eficácia do exame ${ }^{2}$. Pelo EMD é possível observar as estruturas vegetativas dos fungos (parasitismo), em contrapartida, através da cultura, podemos analisar as estruturas reprodutivas, que são essenciais para a correta identificação ${ }^{3}$. A ausência da cultura no exame micológico não permite a identificação da espécie fúngica, e pode ocasionar erros analíticos no exame.

Para salientar a importância do exame cultural no diagnóstico micológico e verificar os possíveis impactos gerados pela não realização desse exame, foram analisados 1603 laudos provenientes do setor de micologia de um laboratório de análises clínicas, com o objetivo de comparar a concordância entre o EMD e o exame cultural, além dos parâmetros de sensibilidade de especificidade das técnicas.

\section{MÉTODOS}

Foi realizado um estudo transversal retrospectivo num laboratório de análises clínicas localizado em Porto Alegre, através da análise de laudos dos exames micológicos realizados entre janeiro de 2018 e maio de 2020. A partir dos resultados obtidos, foi possível dividir as amostras em: EMD negativo com cultura negativa, EMD positivo com cultura positiva, EMD positivo com cultura negativa e EMD negativo com cultura positiva. Como critério de inclusão, foram selecionados os laudos que continham o EMD e a cultura na requisição do clínico. Foram excluídos os laudos com diagnóstico de Malassezia sp., já que esse fungo é identificado somente pelo EMD, pois necessita de um método de cultura específico para o seu crescimento, o que poderia influenciar nos nossos resultados.

Neste laboratório as amostras são coletadas e enviadas ao setor de micologia para a realização do EMD e cultura. Parte da amostra é clarificada com $\mathrm{KOH} 20 \%$ para posterior análise no microscópio óptico, onde é verificada a presença ou ausência de estruturas fúngicas (EMD). O exame cultural é realizado pela inoculação do restante da amostra em uma placa contendo Ágar Batata Dextrose e outra placa contendo Ágar Sabouraud Dextrose com Cloranfenicol, que vai permitir a identificação do fungo. O período de incubação das amostras é de até 21 dias à $25^{\circ} \mathrm{C}$.

A análise estatística foi realizada pelo teste de McNemar, em que o valor de $p<0.01$ foi considerado significativo. O coeficiente de Kappa foi aplicado para verificar o grau de concordância entre as metodologias (EMD e cultura). Os parâmetros de sensibilidade, especificidade, valor preditivo positivo e negativo foram acessados a partir da matriz 2x2 do EMD e cultural.

\section{RESULTADOS}

No período de janeiro de 2018 a maio de 2020 foram realizados 1603 exames micológicos que apresentavam na requisição médica o pedido de EMD e cultura. Os laudos revelaram que a maioria dos casos apresentaram o EMD negativo com cultura positiva ( $36,24 \%)$. Na sequência, $30,87 \%$ dos casos foram de amostras negativas e $25,57 \%$ dos laudos foram positivos para ambos os exames. A minoria dos casos $(7,29 \%)$ apresentou o EMD positivo com cultura negativa (Tabela 1 ).

A relação dos fungos que foram isolados nas amostras de unha, pele, couro cabeludo, cabelo e uretral estão descritos na Tabela 2. Os fungos foram identificados pela cultura positiva, aliada ao EMD positivo ou negativo, com exceção de Malassezia spp., identificada somente pelo EMD.

Tabela 1: Descrição dos laudos dos exames micológicos realizados entre Janeiro de 2018 e Maio de 2020.

\begin{tabular}{lcccc}
\hline & \multicolumn{3}{c}{ Ano } \\
\cline { 2 - 5 } & $\mathbf{2 0 1 8}$ & $\mathbf{2 0 1 9}$ & $\mathbf{2 0 2 0}$ & Total N (\%) \\
\hline EMD Negativo/ Cultura negativa & 210 & 236 & 54 & $\mathbf{4 9 5}(\mathbf{3 0 , 8 7})$ \\
EMD Positivo/ Cultura Positiva & 146 & 227 & 37 & $\mathbf{4 1 0}(\mathbf{2 5 , 5 7 )}$ \\
EMD Positivo/ Cultura Negativa & 11 & 101 & 5 & $\mathbf{1 1 7}(\mathbf{7 , 2 9})$ \\
EMD Negativo/ Cultura Positiva & 316 & 166 & 99 & $\mathbf{5 8 1}(\mathbf{3 6 , 2 4 )}$ \\
Amostras & 473 & 494 & 141 & $\mathbf{1 6 0 3}$ \\
\hline
\end{tabular}

EMD: Exame micológico direto.

(\%) Porcentagem referente ao número total de laudos (1603). 
Tabela 2: Descrição dos fungos isolados em amostras de unha, pele, couro cabeludo, cabelo e uretral.

\begin{tabular}{|c|c|c|c|c|c|}
\hline \multirow[b]{2}{*}{ Isolados } & \multicolumn{5}{|c|}{ Amostra } \\
\hline & Unha & Pele & $\begin{array}{c}\text { Couro } \\
\text { Cabeludo }\end{array}$ & Cabelo & Uretral \\
\hline A. pullulans & 8 & 2 & & & \\
\hline Acremonium spp. & 3 & 2 & & & \\
\hline Alternaria spp. & 1 & & & & \\
\hline Bipolaris spp. & & & 1 & & \\
\hline Candida spp. & 164 & 25 & 1 & & 3 \\
\hline Cladosporium spp. & 1 & 1 & & & \\
\hline Curvularia spp. & 28 & 3 & & & \\
\hline E. floccosum & 1 & & & & \\
\hline Exophialla spp. & 3 & & & & \\
\hline Fusarium spp. & 79 & 5 & & & \\
\hline Geotrichum candidum & 2 & 1 & & & \\
\hline Hortaea werneckii & 1 & 1 & & & \\
\hline Malassezia spp. & & 15 & 1 & & \\
\hline M. canis & 1 & & 3 & & \\
\hline Mucor spp. & 3 & & & & \\
\hline N. gypsea & 1 & & & & \\
\hline Rhizopus spp. & 3 & & & & \\
\hline Rhizomucor spp. & 2 & & & & \\
\hline Rhodotorula spp. & 21 & 14 & 2 & & \\
\hline Scedosporium spp. & 1 & 3 & & & \\
\hline Scytalidium spp. & 34 & 11 & 1 & & \\
\hline Trichophyton spp. & 16 & 4 & & & \\
\hline T. mentagrophytes & 169 & 44 & 3 & 1 & \\
\hline T. rubrum & 148 & 35 & 2 & & \\
\hline T. schoenleinii & 4 & 1 & & & \\
\hline T. verrucosum & 15 & 10 & & & \\
\hline Trichosporon spp. & 53 & 11 & 1 & & \\
\hline Dermatófito/ levedura* & 12 & 3 & & & \\
\hline Dermatófito/ filamentoso* & 9 & & & & \\
\hline Levedura/ filamentoso* & 6 & & & & \\
\hline Fungo ambiental potencialmente patogênico & 7 & 1 & & & \\
\hline Total & 796 & 192 & 15 & 1 & 3 \\
\hline
\end{tabular}

${ }^{*}$ Coinfecção; A. pullulans = Aureobasidium pullulans; E. floccosum = Epidermophyton floccosum; $M$. canis = Microsporum canis; $N$. gypsea = Nannizzia gypsea; T. mentagrophytes = Trichophyton mentagrophytes; $T$. rubrum = Trichophyton rubrum; $T$. schoenleinii = Trichophyton schoenleinii; $T$. verrucosum $=$ Trichophyton verrucosum .

De acordo com os critérios apresentados por $\mathrm{McHugh}^{4}$, o grau de concordância entre o EMD e cultural encontrado em nosso estudo foi fraco, com valor de Kappa $=0,196$, o erro padrão do coeficiente Kappa foi de 0,020 , perfazendo um intervalo de confiança $95 \%$ de 0,157 a 0,235 . Os parâmetros de sensibilidade, especificidade, valor preditivo positivo e negativo, para o EMD e cultural podem ser visualizados na Tabela 3. Consideramos um resultado falso positivo para o EMD, quando o mesmo foi negativo e a cultura foi positiva. Para a cultura, um resultado falso positivo foi considerado quando foi observado crescimento de fungo ambiental contaminante e EMD negativo.

A comparação entre as duas metodologias através do teste de McNemar mostrou que o valor bicaudal foi menor que 0,0001 , pelos critérios convencionais essa diferença estatística é considerada extremamente significante. A equivalência Chi-quadrado foi de 307,119 com 1 grau de liberdade. A diferença entre as metodologias estudadas é significativa para um valor de $\mathrm{P}<0.01$. 
Quatrin et al.

Tabela 3: Determinação da sensibilidade, especificidade, valor preditivo negativo e positivo do EMD e cultura.

\begin{tabular}{ccccc}
\hline Método de diagnóstico & Sensibilidade & Especificidade & VPP & VPN \\
\hline EMD & $38,78 \%$ & $81,04 \%$ & $77,80 \%$ & $43,59 \%$ \\
Cultura & $89,44 \%$ & $94,16 \%$ & $96,97 \%$ & $81,04 \%$ \\
\hline
\end{tabular}

EMD: Exame micológico direto; VPP: Valor preditivo positivo; VPN: Valor preditivo negativo.

A sensibilidade é a probabilidade de um teste dar positivo na presença do agente etiológico, isto é, avalia a capacidade do teste detectar crescimento quando ele deve ocorrer. Já a especificidade é a probabilidade de o teste dar negativo na ausência do agente etiológico, ou seja, o teste se apresentar negativo quando ele deve o ser. A partir dessas métricas podemos identificar de melhor forma os exames realmente positivos e negativos (especificidade e sensibilidade) ${ }^{5}$.

O valor preditivo positivo (VPP) é a proporção de verdadeiros positivos entre todos os indivíduos com exame positivo, enquanto o valor preditivo negativo (VPN) é a proporção de verdadeiros negativos para todos os testes negativos. Dessa maneira, quanto mais sensível é um teste, maior o seu VPN, e quanto mais específico é o teste maior o VPP. As curvas ROC (receiver operator characteristic curve) são uma forma de representar a relação, normalmente antagônica, entre a sensibilidade e a especificidade de um teste diagnóstico quantitativo ${ }^{5}$.

\section{DISCUSSÃO}

A diferença significativa entre as metodologias estudadas demonstra que a escolha do método de diagnóstico influencia diretamente no resultado liberado pelo analista. Contrariando achados na literatura que apontam o EMD como mais relevante quando comparado aos demais meios diagnósticos, nossos resultados demonstram que há maior confiabilidade quando é utilizada a cultura do microrganismo de interesse. O exame cultural sempre deve ser realizado em conjunto com o EMD, pois além de reduzir os riscos de resultados falso negativos, apresenta maior especificidade ${ }^{6,7}$. O grau de concordância fraco entre os dois exames observado na nossa investigação foi inferior ao que relatam algumas publicações ${ }^{3,8}$, e está relacionado com as diferenças de sensibilidade entre as técnicas. Observamos uma grande diferença de sensibilidade entre as metodologias, de quase $90 \%$ para a cultura e $40 \%$ para o EMD, contrapondo os achados na literatura que apontam o EMD como mais sensível ${ }^{8,9}$. No estudo de Zanardi et al. ${ }^{7}$, a sensibilidade e a especificidade da cultura, $84,6 \%$ e $100 \%$ respectivamente, foi semelhante ao encontrado em nosso estudo. Em contrapartida, o EMD apresentou sensibilidade elevada (100\%), divergindo novamente dos nossos achados.

Entre os 1603 laudos analisados, 1108 apresentaram confirmação micológica, em sua maioria através do exame cultural. Observamos que 581 amostras poderiam ter sido laudadas erroneamente como negativas, caso a cultura não fosse realizada. Weinberg et al. ${ }^{6}$ relata que o EMD possui uma porcentagem de falsos negativos que varia entre 5 e $15 \%$, provavelmente pela dificuldade de visualização das células fúngicas dispersas na lâmina, sendo que nosso estudo revelou uma porcentagem superior a $50 \%$. Além da habilidade do analista, a qualidade da amostra influencia diretamente no resultado do EMD e do exame cultural, como amostras insuficientes ou coletadas distalmente ao crescimento fúngico $0^{6,10}$.

Um laudo micológico negativo pode ser erroneamente confundido com um falso negativo, o que gera descredito no clínico. Para minimizar esse risco, Meireles et al. ${ }^{11}$ sugerem que três amostras consecutivas sejam coletadas em intervalos de 2 a 5 dias, aumentando dessa forma a precisão do diagnóstico. A distribuição irregular dos fungos em lesões, ou amostras com extrema queratinização - como em casos de onicomicose - que dificultam a coleta, também podem gerar uma diminuição ou até mesmo ausência de esporos fúngicos na amostra ${ }^{11}$, contribuindo para um resultado errôneo.

Levantamentos recentes baseados em laudos micológicos são fundamentais para evidenciar a importância do exame micológico clássico, composto pelo EMD e cultural, ambos com as suas particularidades. Exames que possuem elevada sensibilidade são bons indicadores, e funcionam bem para testes de triagem quando queremos afastar um diagnóstico, já os exames específicos são confirmatórios, e esses dois parâmetros observados em nosso estudo para o exame cultural apontam a sua relevância para um diagnóstico mais fidedigno.

Em nosso estudo o exame cultural apresentou maior sensibilidade e especificidade, e quando aliado ao EMD torna-se uma potente ferramenta de diagnóstico. A experiência do analista e a qualidade da amostra são fatores que podem influenciar no resultado dessas técnicas. Somente através da cultura é possível identificar precisamente o agente patogênico a nível de espécie. A realização destes exames em conjunto proporciona um resultado fidedigno, minimizando possíveis erros analíticos, como a ocorrência de falso negativos.

\section{Conflitos de Interesse}

Os autores declaram não haver conflito de interesse. 


\section{REFERÊNCIAS}

1. Lachaud L, Sasso M, Rispail P, Bourgeois N. Diagnostic biologique des onychomycoses: examen direct après coloration PAS simplifiée. J Mycol Méd. 2014;24:279-86.

2. Chauvin MF. Examen mycologique en dermatologie. Ann Dermatol Venereol. 2018;145:623-32.

3. Azambuja CVA, Pimmel LA, Klafke GB, Xavier MO.

Onychomycosis: clinical, mycological and in vitro susceptibility testing of isolates of Trichophyton rubrum. An Bras Dermatol. 2014;89:581-6.

4. McHugh ML. Interrater reliability: the kappa statistic. Biochem Med. 2012;22:276-82.
5. Trajman A, Luiz RR. McNemar x2test revisited: comparing sensitivity and specificity of diagnostic examinations. Scand J Clin Lab Invest. 2008;68:77-80.

6. Weinberg JM, Koestenblatt EK, Tutrone WD, Tishler HR, Najarian L. Comparison of diagnostic methods in the evaluation of onychomycosis. J Am Acad Dermatol. 2003;49:193-7.

7. Zanardi D, Nunes DH, Pacheco AS, Tubone MQ, Souza JJ Filho. Evaluation of the diagnostic methods of onychomycosis. An Bras Dermatol. 2008;83:119-24.

8. Souza PR, Vettorato G, Pinto GM, Duquia RP, Amaro TG, Almeira Junior HL, et al. Concordance between direct microscopy and fungical culture for the diagnostic of feet's onychomycosis. An Bras Dermatol. 2012;87:157-9.
9. Veasey JV, Nappi F, Zaitz C, Muramatu LH. Descriptive analysis of mycological examination of patients with onychomycosis treated in private practice. An Bras Dermatol. 2017;92:134-6.

10. Panasiti V, Borroni RG, Devirgiliis V, Rossi M, Fabbrizio L, Masciangelo R, et al. Comparison of diagnostic methods in the diagnosis of dermatomycoses and onychomycoses. Mycoses. 2006;49:26-9.

11. Meireles TE, Rocha MF, Brilhante RS, Cordeiro RA, Sidrim JJ. Successive mycological nail tests for onychomycosis: a strategy to improve diagnosis efficiency. Braz $J$ Infect Dis. 2008;12:333-7.

Recebido: 25 out, 2020 Aceito: $23 \mathrm{dez}, 2020$ 